\title{
NICMOS observations of the HDF
}

Rodger I. Thompson, Ray J. Weymann, and Lisa Storrie-Lombardi

Citation: AIP Conference Proceedings 470, 113 (1999); doi: 10.1063/1.58593

View online: $\mathrm{http}: / / \mathrm{dx}$.doi.org/10.1063/1.58593

View Table of Contents: http://aip.scitation.org/toc/apc/470/1

Published by the American Institute of Physics 


\title{
NICMOS Observations of the HDF
}

\author{
Rodger I. Thompson*, Ray J. Weymann ${ }^{\dagger}$, Lisa Storrie-Lombardi ${ }^{\dagger}$ \\ * Steward Observatory, University of Arizona, Tucson, AZ 85721 \\ ${ }^{\dagger}$ Carnegie Observatories, 813 Santa Barbara St., Pasadena, CA, 91101-1292
}

\begin{abstract}
This paper presents initial results and performance levels from the Near Infrared Camera and Multi-Object Spectrometer (NICMOS) observations of the Hubble Deep Field (HDF). These observations represent the deepest view of individual objects yet obtained with photometric colors of some objects indicating redshift values greater than 6. These observations add significant value to the previous optical observations of the HDF with the Wide Field and Planetary Camera II (WFPC II).
\end{abstract}

\section{INTRODUCTION}

A major consideration in the instrument design and initial scientific program of NICMOS was the realization that high sensitivity imaging at near infrared wavelengths $(0.8-2.5 \mu \mathrm{m})$ offers a deeper view of distant galaxies than optical measurements that are limited by hydrogen continuum and Lyman line absorption of the galactic light. In particular the wide field NICMOS camera 3 was the best mode for deep observations. The minimum background for the NICMOS instrument occurs at $1.6 \mu \mathrm{m}$ at the intersection of the zodiacal light reflection that rises toward shorter wavelengths and the thermal emission from the HST mirrors that rises toward longer wavelengths. This minimum background region is exploited by the wide band filter $\mathrm{F} 160 \mathrm{~W}$ centered on the $1.6 \mu \mathrm{m}$ wavelength with a spectral width of approximately $0.4 \mu \mathrm{m}$.

The advent of original HDF observations [7] offered an excellent opportunity to combine deep near infrared and optical imaging over a limited area of the sky. The NICMOS Guaranteed Time Observation (GTO) orbit allocation contributed 127 orbits for the conduct of this program. The majority of the orbits were for imaging but a few orbits utilized the slitless grism mode for wide field spectroscopy. This paper will only consider the imaging mode observations.

One of the main products of the WFPC II HDF observations is a catalog of the photometric properties of all of the detected sources in the region [7]. A similar catalog of the NICMOS sources is now available [4]. Since this publication contains

CP470, After the Dark Ages: When Galaxies were Young (the Universe at $2<z<5$ ), edited by Stephen S. Holt and Eric P. Smith

(C) 1999 The American Institute of Physics 1-56396-855-X/99/\$15.00 
the detailed images they will not be repeated here.

\section{OBSERVATIONAL PARAMETERS}

The implementation of a high sensitivity, low flux limit imaging program requires careful selection of the observational parameters. These parameters include the camera selection, filters, detector read out modes, and allocation of orbits between imaging and spectroscopic modes. Also included is the exact spatial positioning of the observations.

\section{Camera and Filter Selection}

As indicated in the introduction the NICMOS camera 3 was designed with deep imaging as an important priority and is the obvious choice for the observations. Although diffraction limited, cameras 1 and 2 have too limited a field of view for effective areal coverage. The 0.2 arc second pixel size of camera 3 was chosen to be dominated by the natural zodiacal background over the dark current while maintaining a relatively high resolution. Camera 3 was therefore the camera of choice.

Also, as indicated in the introduction, the F160W is designed for low background deep imaging and is therefore an obvious filter choice. A second filter is required to provide color information on high red shift sources that have little or no flux in the optical bands. Without this second filter it is not possible to discriminate between high redshift and heavily extincted galaxies. The exponentially rising thermal flux at longer wavelengths precluded picking a longer wavelength filter. The selected second filter is the wide band F110W filter centered at $1.1 \mu \mathrm{m}$. Although this filter has significant spectral structure it provides greater signal to noise observations and higher spectral discrimination than any other available filter.

\section{Spatial Distribution of the Observations}

Since the field of view the NICMOS camera 3 is significantly less than the WFPC II field there is a choice between coverage of the whole HDF at lower signal to noise or concentration on one area to obtain the deepest possible image. Since our main interest is in high redshift objects beyond the reach of the optical HDF we chose to concentrate on only one area of the HDF. Selection of the particular area was governed in part by the grism observations. These observations require an area free of large bright galaxies whose spectra would overlap large areas of the detector. For this reason we chose our area to be roughly centered on chip 4 of the WFPC image, an area relatively sparse in large galaxies. The orientation of the image was dictated by the scheduling of the observations and available guide stars rather than by scientific criteria. 
Once the choice of field is made the exact positioning of the individual images is important. These choices affect the total area covered, the ability to form background images for subtraction and the reduction of artifacts due to pixel to pixel variations in response. Production of the background image requires images separated by enough distance that sources do not predominantly fall on the same group of pixels. High signal to noise on the other hand requires that most of the region is well covered which argues for short steps between images. The number of orbits allocated to the total program must also be in a reasonable range.

Our choice for this program was a $7 \times 7$ rectangular grid requiring 49 orbits for each filter. Within each orbit there are three integrations each of which is offset from each other by a small step much smaller than the space between grid points. Our orientation placed the field at an approximately 30 degree angle to the original HDF field, therefore, we chose a grid step of 0.918 arc seconds in the $\mathrm{X}$ direction and 1.523 arc seconds in the $\mathrm{Y}$ direction to produce a slightly rectangular observation grid. Three integrations were taken at each grid point which consumed one orbit. The three integrations were dithered by 0.408 arc seconds in the $\mathrm{X}$ and $\mathrm{Y}$ direction so that all integrations had each pixel on a different portion of sky. This resulted in 147 integrations per filter and a total integration time per filter of $1.3 \times 10^{5}$ seconds.

\section{A Areal Coverage}

The total area covered by at least one image is a rectangle of 56.91 by 61.39 arc seconds and the area covered by all integrations is 46.85 by 43.01 arc seconds. The image area covered by the listed catalog sources is 49.19 by 48.53 arc seconds. This choice was a subjective judgement on the region containing good signal to noise not compromised by a low number of integrations. Since the quantum efficiency of the NICMOS detectors varies significantly across the array the appropriate area of coverage was not a straightforward calculation.

\section{B Detector Parameters}

From the selection of standard readout patterns we chose the SPARS 64 detector readout for all of the integrations. This pattern has three readouts closely spaced then a readout every 64 seconds. With the number of readouts set to 17 $(\mathrm{NSAMP}=17)$ the total integration time was 896 seconds for each image. This choice provided three separate integrations per orbit and enough individual readouts to allow excellent removal of cosmic ray hits. Before the observations it was thought that the integration time would produce a background noise from the zodiacal light roughly equal to the read noise. The zodiacal light at the location of the HDF turned out to be significantly lower than expected $(0.55 \mathrm{e} / \mathrm{sec})$, therefore, each integration was read noise limited. 

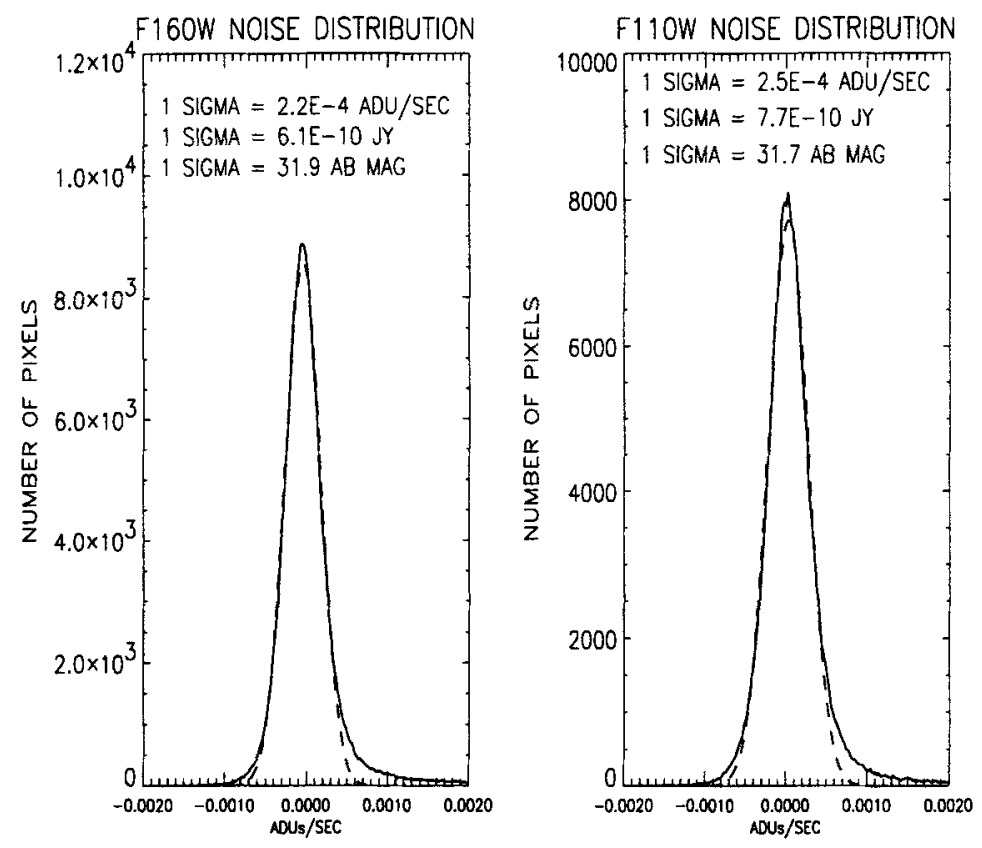

FIGURE 1. Histogram of pixel values for the F160W and F110W images. The 1 sigma values must be multiplied by a factor of 1.9 to account for the correlation of drizzled pixels

\section{Performance Levels}

The basic result is that the image signal to noise ratio is equal to the ratio calculated from the known read noise, dark current, quantum efficiency and observed sky background. This means that we did not have contributions from previously unknown noise sources or systematic effects that compromised the signal integrity. Aggressive scheduling of the HDF observations by STScI resulted in few integrations that were contaminated by cosmic ray persistence due to recent passage through the South Atlantic Anomaly (SAA). The net usable integrations were 122 in the F160W filter and 118 in the F110W filter.

Figure 1. shows the distribution of flux levels per pixel in ADUs per second for both the F160W and F110W filter. The pixels referred to in this figure are the drizzled [2] pixels of size 0.1 by 0.1 arc seconds instead of the physical pixel size of 0.2 by 0.2 arc seconds. Since the drizzeling process produces correlated noise the numbers in the one sigma numbers in the figure must be multiplied by a factor of 1.9 [7] to produce the proper 1 sigma noise number. The one pixel sigmas are then $1.5 \times 10^{-9} \mathrm{Jy}\left(31.0 \mathrm{AB}\right.$ mag) for the F110W filter and $1.2 \times 10^{-9} \mathrm{Jy}(31.2 \mathrm{AB}$ $\mathrm{mag})$ for the F160W filter.

Each of the 896 second integrations that did not have significant residual cosmic 

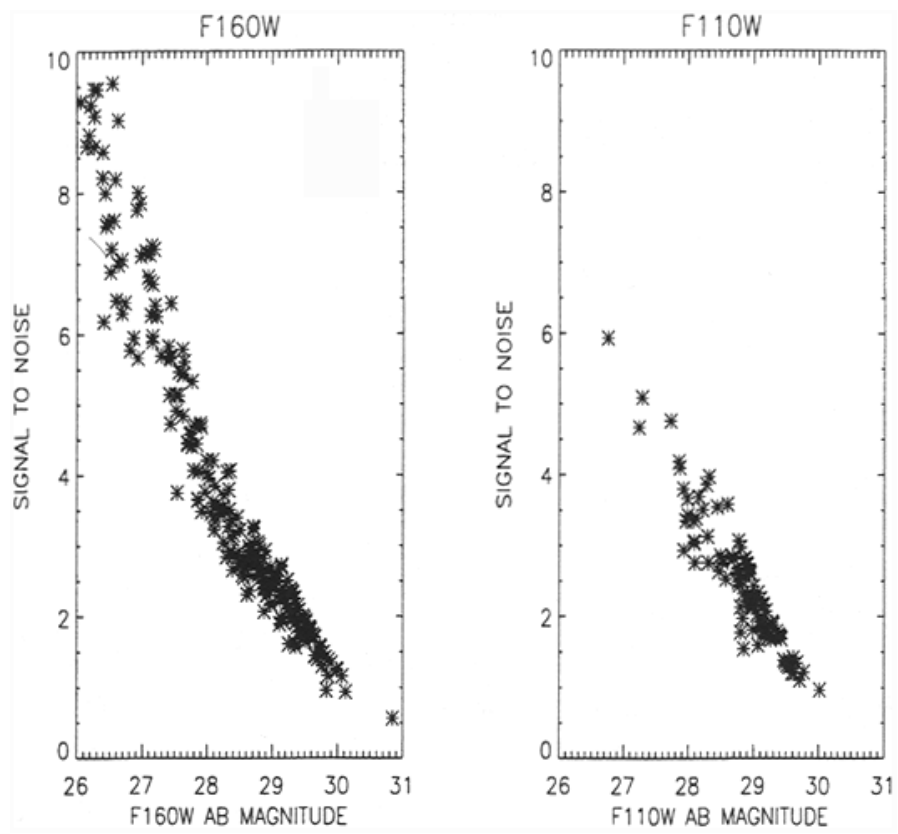

FIGURE 2. Distribution of the signal to noise ratio versus magnitude for the detected sources. Only sources with signal to noise ratios less than 10 are plotted.

ray noise from a SAA passage was used in the final mosaic image. Histograms of the individual images displayed the expected signal to noise ratio from the parameters given above. The final histogram displayed in Fig. 1 had a FWHM reduced by a factor of the square root of the number of integrations from the average individual integration FWHM. This is further indication that systematic errors did not dominate in the final image. It should be noted that the single pixel error is not the expected error level for galaxy detection. Since galaxies are spread over several pixels they will have a higher error level. Figure 2 shows the distribution of signal to noise versus magnitude for the detected sources. Some of the spread in this plot, particularly at higher signal to noise levels is due to sources of the same magnitude spread over a different number of pixels.

\section{Results of the Observations}

One of the main results of these observations is a catalog of sources with measured signal to noise levels greater or equal to 2.5 [4]. The electronic version of that reference contains an additional catalog of detected sources with signal to noise ratios less 2.5. One of the main purposes of the additional catalog is to provide 


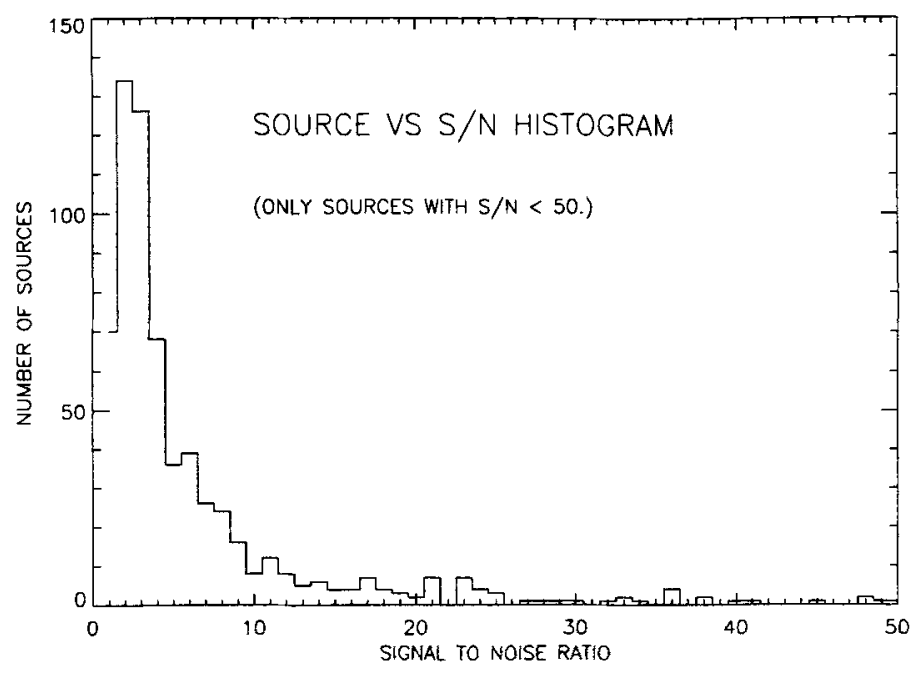

FIGURE 3. Histogram of the number of sources versus signal to noise ratio. The figure has been truncated at a signal to noise level of 50 for clarity.

information of the limits of source level detection for researchers comparing results from other wavelength regions. Figure 3 shows a distribution of sources versus signal to noise in 1.0 sigma bins. The figure is cut at a signal to noise ratio of 50 for clarity, although there are several sources with higher signal to noise ratios.

The NICMOS catalog does contain correspondence information with the WFPC II catalog [7] which gives the identification and distance in arc seconds to the nearest WFPC II source. A NICMOS and WFPC II source are considered to correspond if their separation is less than 0.25 seconds of arc. In some cases differing morphology between the infrared and optical regions results in false noncorrespondance in a source.

In the following results we will refer only to those sources that appear in the catalog of sources with signal to noise ratios greater than or equal to 2.5. This catalog contains 342 separately listed objects.

\section{A High Redshift Objects}

Of the 342 objects in the catalog, 107 objects have no corresponding WFPC II identification. A few of these are due to the differences in infrared and optical morphology mentioned above. Most of these objects are highly reddened galaxies which are too faint at optical wavelengths to be detected in the original HDF observations. A few of these galaxies, however, may be truly high redshift galaxies. These galaxies have little or no flux in the WFPC II F814W filter but have flat 
or blue color in the NICMOS F110W - F160W bands. It is presumed this flux distribution is due to the Lyman limit falling in the F814W band and the young blue galaxy UV flux falling in the NICMOS F110W and F160W bands. It is possible that at low flux levels this could also be caused by the 4000 Angstrom break falling in the F814W band but in most cases the lack of flux in the WFPC II F606W filter is inconsistent with this interpretation. At present about 10 to 20 objects are high redshift candidates. One of these objects is NICMOS source 184.0 which corresponds to WFPC source 4-473. This source has a spectroscopicly confirmed redshift of 5.61 [6]. It has low F814W flux, but enough to be detected by WFPC, and a blue F110W - F160W color. It is bright enough in the infrared that even if it were dimmed to a redshift of 10 it would still be visible in the NICMOS images. It is strong evidence that if there are sources of this type at redshifts greater than 5.6 they are visible in the NICMOS image. After a redshift of 10 almost all of the flux lies in the F160W band and these sources are indistinguishable from source with very high extinction. Detailed investigation of high redshift candidates is the subject of a paper in preparation [5].

\section{B Small Blue Objects}

Another result of inspection of the NICMOS images concerns the nature of the numerous small blue objects that appear in the original WFPC II HDF images. To date these objects have generally assumed to be small galaxies or perhaps fragments of galaxies that will merge at a later date. They can not be extremely distant objects since they have a significant amount of blue flux. Two point correlation studies [1] on the other hand indicate they are spatially more correlated than would be expected for a random distribution of galaxies.

Some clues to the nature of these sources comes from the inspection of WFPC source 4-378. This is clearly a spiral galaxy with bright star formation regions in its spiral arms. This source corresponds to NICMOS source 124.0. In the infrared the star formation regions are dominated by the old stellar population that clearly shows the nucleus of the galaxy, offset from the star formation regions. This source may be a Rosetta stone for the identification of many of the small blue sources. If 4-378 were somewhat fainter only the bright blue star formation regions would be visible in the optical images, similar to the isolated small blue objects. If fainter in the infrared only the dominant red nucleus would still be visible nearby but not coincident with the small blue objects. In fact when the optical and infrared images are overlayed small infrared objects are often found very near individual or groups of small blue objects. The separation distance of the objects are less than the radii of the several $z=1$ elliptical and spiral galaxies in the field. Since in most cosmologies the angular size of galaxies either reaches a minimum or very slowly decreases past $z=1$ the grouping of infrared and blue objects fall within the region

of a single galaxy. A logical conclusion from these observations is that many of the small blue objects in the optical HDF image may simply be bright star formation 
regions in a dim galaxy whose nuclear region appears in the infrared image. Details of this analysis will appear in a forthcoming paper [3].

\section{Conclusions}

The NICMOS images of part of the original HDF field gives us the most distant view of individual galaxies in the Universe to date. It provides also provides images of distant galaxies at rest wavelengths in the optical region where galaxies have been well studied. Several high redshift candidates have been identified but spectroscopic follow up will be difficult due to the faintness of the images. In addition we find very suggestive evidence that many of the small blue objects in the WFPC HDF image may be star formation regions in a much larger underlying galaxy.

As with the original HDF image most of the scientific discovery will come with detailed quantitative investigations. Many of these are underway and will come to fruition in the near future. One of the keys to these studies is the combination of the optical and infrared data from HST and data at many different wavelengths from ground based and other space based observatories.

\section{Acknowledgements}

A very large group of people contributed to the success of the NICMOS HDF observation project. The entire NICMOS Instrument Definition Team contributed both the expertise to make NICMOS a reality and the large number of orbits from the GTO program needed to carry out the observations. We thank Mark Dickinson and Andy Fructher for their help with the reduction and mosaic techniques for the data analysis. Andy Lubenow spent many hours optimizing the observational techniques. Chris Conner and the Lockheed group made an extraodinary effort to keep our pointing accurate during our single guide star integrations. Zolt Levay provided the combined NIMOS and WFPC color image. The work was supported by NASA grant NAG 5-3043 and the observations were obtained with the NASA/ESA Hubble Space Telescope Science Institute managed by the Association of Universities for Research in Astronomy Inc. under NASA contract NAS5-26555.

\section{REFERENCES}

1. Colley, W.N., Gnedin, O.Y., Ostriker, J.P., and Rhoads, J.E. 1997, Ap. J., 488, 579.

2. Fruchter, A.S. and Hook, R.N. 1997, in Applications of Digital Image Processing XX, Proc. SPIE, Vol. 3164, ed. A.Tescher, pp 120-125.

3. Storrie-Lombardi, L., Weymann, R.J., and Thompson, R.I. 1999, in preparation.

4. Thompson, R.I., Storrie-Lombardi, L.J., Weymann, R.J., Rieke, M.J., Schneider, S., Stobie, E., and Lytle, D. 1999, A.J., in press.

5. Weymann, R.J., Thompson, R.I., \& Storrie-Lombardi, L. 1999, in preparation. 
6. Weymann, R.J., Stern, D., Bunker, A., Spinrad, H., Chaffe, F.H., Thompson, R.I., and Storrie-Lombardi, L., 1998, Ap.J. (Letters), 505, L95.

7. Williams, R.E., Blacker, B., Dickinson, M., Dixon, W.V.D., Ferguson, H.C., Fruchter, A.S., Giavalisco, M., Gilliland ,R. L., Heyer, Inge, Katsanis, R., Levay, Z., Lucas, R.A., McElroy, D.B., Petro, L., Postman, M., Adorf, H-M, and Hook, R.N. 1996, A.J. 112, 1335. 